\title{
Column size effects of DER fluids
}

\author{
Tianyu Zhao and H. R. Ma \\ Institute of Theoretical Physics, Shanghai Jiao Tong University, Shanghai, 200240, China
}

\begin{abstract}
The static yield stress of dielectric electrorheological(DER) fluids of infinite column state and chain state are calculated from the first principle method. The results indicate that the column surface contributions to ER effects is very small and both states will give correct results to the real DER fluids.
\end{abstract}

PACS numbers: 83.80.Gv,83.60.La,41.20.Cv

In the past two decades, there has been a renewed interest in the researches of Electrorheological (ER) fluids. This is partly stimulated by the potential and practical applications of this material, and partly by the desire of understanding of the physics of many complex systems to which the ER fluids is belong. The ER fluids are a class of materials whose rheological properties are controllable by the application of an electric field. It is generally made up of solid particles dispersed in a liquid. Due to the large varieties of electric properties of both the solid particles and liquids, and varieties of shape, size distributions of solid particles, the ER mechanism is still not well understood in general. In this paper we will focus on the simplest type of ER fluids: uniformly sized, solid dielectric spheres dispersed in a liquid. Both the solid and the liquid components are assumed to follow linear electrostatic response under an applied field. This type of ER fluids is denoted as the dielectric electrorheological (DER) fluids [1]. It was predicted by Halsey et al[2] that in the DER fluids the solid particles aggregate to form columns under electric field, Based on induced dipole interactions of solid particles, Tao et al[3] argued that the structure within columns is body centered hexagonal(BCT). These predictions were confirmed by accurate electrostatic calculations with different methods [1, 5, 6, 7, 8]. From calculations of Ma et

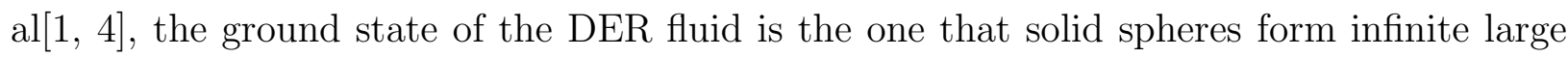
columns and the structure within the column is BCT. However, experiments usually saw chains of solid particles under weak fields and small columns under strong fields, the size of the columns may be determined by the dynamic process of the aggregation and is still poorly understood. On the other hand, first principle calculations based on the assumption 
of infinite large columns gave quantitative agreement to the well controlled experiment without adjustable parameters[罒]. It was argued in [1] based on the calculation of electrostatic free energy that the influence of column size to static yield stress is small. We will give, in this paper, a comparison of static yield stress in two extreme cases of smallest column size(chain state) and infinite column state. The results of our calculation indicate that the column size effect is indeed small, as asserted in [1].

The method of calculation in this study is the first principle method developed by Ma et al [1, 团, based on the Bergman-Milton representation of effective dielectric constant of a composites, and outlined in the following.

Consider a DER model system consisting of spherical solid particles of radius $\mathrm{R}$ and dielectric constant $\epsilon_{1}$ dispersed in a fluid characterized by $\epsilon_{2}$. The equation for the electrostatic potential $\phi(r)$ is :

$$
\nabla \cdot[\epsilon(\mathbf{r}) \nabla \phi(\mathbf{r})]=0,
$$

where the dielectric constant $\epsilon(\mathbf{r})=\epsilon_{2}[1-\eta(\mathbf{r}) / s], s=\epsilon_{2} /\left(\epsilon_{2}-\epsilon_{1}\right)$ is the only material parameter in the problem, and $\eta(\mathbf{r})$ is the characteristic function of the solid component, defined as having the value 1 at those spatial points occupied by the solid particles, and zero otherwise. Following Bergman and Milton[9], the solution of Eq.(1) directly yields the effective dielectric constant $\bar{\epsilon}_{z z}$ of the system,

$$
\bar{\epsilon}_{z z}=\epsilon_{2}\left(1-\frac{1}{s V} \int d \mathbf{r} \eta \frac{\partial \phi(\mathbf{r})}{\partial z}\right)=\epsilon_{2}\left(1-\sum_{n} \frac{f_{n}}{s-s_{n}}\right)
$$

where $\phi=[1-\Gamma / s]^{-1} z$ is the formal solution to Eq.(1), with

$$
\Gamma=\int d r^{\prime} \eta\left(\mathbf{r}^{\prime}\right) \nabla^{\prime} G\left(\mathbf{r}, \mathbf{r}^{\prime}\right) \cdot \nabla^{\prime}
$$

and $G\left(\mathbf{r}, \mathbf{r}^{\prime}\right)=1 /\left(4 \pi\left|\mathbf{r}-\mathbf{r}^{\prime}\right|\right)$ being Green's function for the Laplacian operator, $V$ denotes the sample volume. $s_{n}$ is the eigenvalue of the $\Gamma$ operator and $f_{n}$ related to the eigenfunction of $\Gamma$. Both $s_{n}$ and $f_{n}$ are determined only by the microstructure of the material and independent of the material parameters. Detailed description of the effective dielectric constant calculation

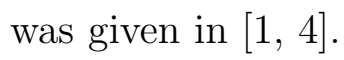

Since the free energy density of the system in the high-field regime is given by $f=$ $-\bar{\epsilon}_{z z} E^{2} / 8 \pi$, the ground state structure is determined by maximizing $\bar{\epsilon}_{z z}$ with respect to the coordinates of the solid particles. For ER fluids, a great deal of experiments and calculations 
show that the BCT structure is the optimal structure. To calculate the static yield stress, one just needs to perturb the system away from the ground state by applying a shear deformation to the system that is perpendicular to the applied field direction. The shear distortion consists of two parts, the $c$ axis of the BCT structure is both tilted by an angel $\theta$ with respect to the field as well as elongated, and the $a b$ plane is shrunken uniformly to stabilize the system by hard core repulsion of spheres. The volume fraction of solid spheres in the structure is also $\theta$ dependent under shear, given by $p_{0}(\theta)=4 \pi \cos ^{2} \theta / 3\left(8 \cos ^{2} \theta-2\right)$. The free energy density of the system can be writes as :

$$
f=-\left(\bar{\epsilon}_{c c} \cos ^{2} \theta+\bar{\epsilon}_{a a} \sin ^{2} \theta\right) \frac{E_{0}^{2}}{8 \pi}
$$

where $E_{0}$ denotes the applied electric field, along $z$ direction. $\bar{\epsilon}_{c c}$, the $c c$ component of the effective dielectric tensor of the system, is accurately related to the $c c$ component of effective dielectric constant within the tilted $\mathrm{BCT}$ structure, $\bar{\epsilon}_{c c}^{\infty}$, by the relation

$$
\bar{\epsilon}_{c c}=\frac{p}{p_{0}} \bar{\epsilon}_{c c}^{\infty}+\left(1-\frac{p}{p_{0}}\right) \epsilon_{2}
$$

where $p$ denotes the volume fraction of spherical solid particles of the system. While $\bar{\epsilon}_{a a}$ may be approximated by the Maxwell-Garnett formula [10]:

$$
\bar{\epsilon}_{a a}=\frac{\bar{\epsilon}_{a a}^{\infty}\left(1+p / p_{0}\right)+\epsilon_{2}\left(1-p / p_{0}\right)}{\epsilon_{a a}^{\infty}\left(1-p / p_{0}\right)+\epsilon_{2}\left(1+p / p_{0}\right)}
$$

with $\bar{\epsilon}_{a a}^{\infty}$ the $a a$ component of the effective dielectric constant within the tilted BCT structure. Here $a$ and $c$ are the crystallography coordinates of the tilted BCT structure.

The stress-strain relation is given by $\partial f / \partial \theta$. To calculate the stress from effective dielectric constant, the effective dielectric constant at a mesh of $\operatorname{strain}(\theta)$ was generated, then the differentiation was numerically calculated by the third order spline interpolation method. The static yield stress is defined as the maximum stress as function of strain.

In the case of chain state, the starting structure is assumed to be single aggregated chains form a regular two dimensional(2D) lattices, the 2D lattice constant is chosen according to the prescribed over all volume fraction $p$. By shearing the system, the spheres within each chain are assumed uniformly separated. $\bar{\epsilon}_{c c}$ and $\bar{\epsilon}_{a a}$ are calculated by method described above. It should be noted that the distorted chain states are unstable to the formation of small aggregated short chains by electrostatic interaction. However, we use these seemingly unphysical state for the following two reasons. First, if we use small columns of 2 or more 


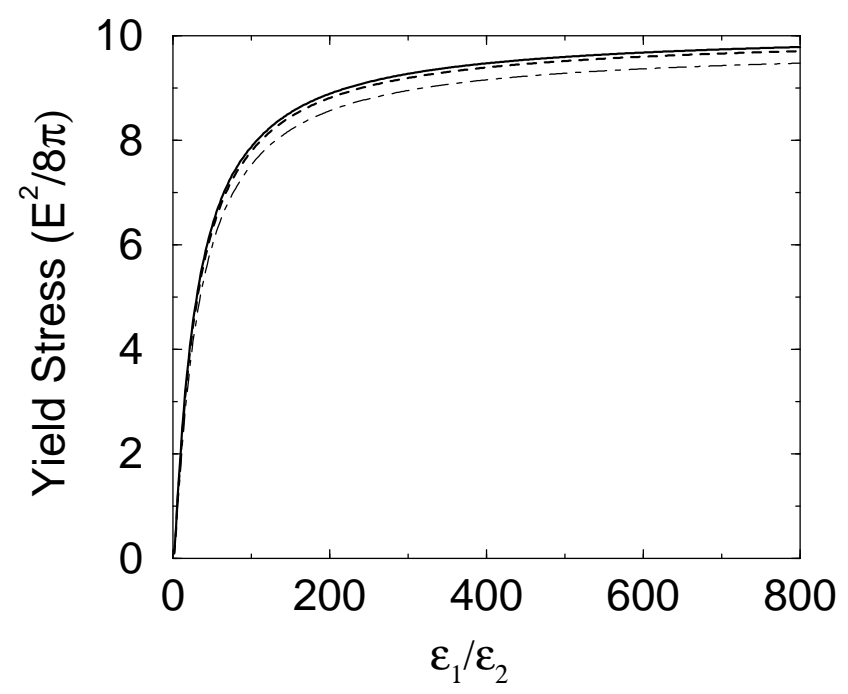

FIG. 1: Comparison between the static yield stress of chain states of simple square lattice(dashed line), the expanded BCT(solid line) and the triangular lattice(dot-dashed line), $\delta=0.01$.

chains, the distorted structure can be stabilized by hard core repulsions of spheres, and the single chain is a limiting case of small columns. Second, we study here only the electrostatic force after distortion, we may think that the system is dielectric particles trapped in a gel so that the structure is stabilized. Since there is no phase separation in the chain state, the average procedure described by Eq. (4) and Eq. (5) are not needed. We did calculations with simple square lattice, the square lattice with neighbour chains shifted by the sphere radius (the expanded BCT) and the triangular lattice. The results are showing in figure 1. All lattices basically give the same results, the largest difference is about $3.1 \%$.

In our calculation, we choose $\epsilon_{2}$ to be 2.5 while $\epsilon_{1}$ ranging from 2.5 to 2000 . The static dielectric constant depends not only on $\epsilon_{1}, \epsilon_{2}$ and the volume fraction, but also sensitively on the closest distance of two nearest spheres, characterized by $\delta$, related to the center to center distance of two nearest spheres $d$ by

$$
d=2 R(1+\delta)
$$

Figure 2 and figure 3 are plots of the calculated static yield stresses for the two cases with different $\delta$. As expected, the static yield stress of the infinite column state is always larger then the chain state. The important part of the figures is that the dependence of static yield stress on the ratio of the dielectric constant $\epsilon_{1} / \epsilon_{2}$ is very similar. The difference 


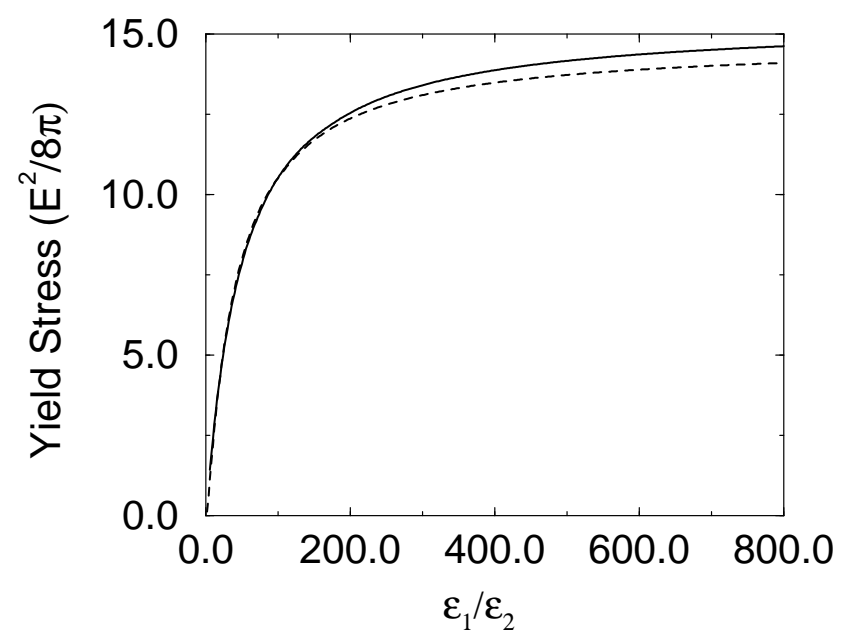

FIG. 2: Comparison between the static yield stress of infinite column state(solid line) and the expanded BCT chain state(dashed line). The volume fraction of the system is $0.2, \delta=0.005$.

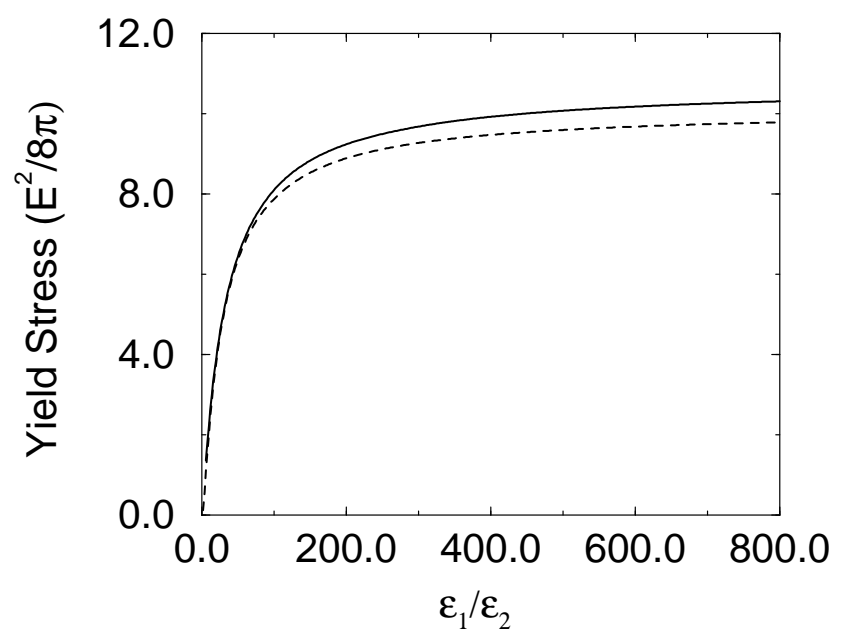

FIG. 3: Same as figure 2, with $\delta=0.01$

between the two is very small. The largest difference was found on the case of smallest $\delta$ and largest dielectric constant mismatch, which is only $3.75 \%$.

As pointed out in $[\mathbb{1}$, the surface energy of columns in DER fluids is very small compared to the bulk electrostatic free energy. Our calculation indicates that not only the surface energy is small, its contribution to the static yield stress is also very small. Attempts to improve the electrorheological effects by increase the column size is extremely limited, at least in the DER case. This is why the theory give excellent agreement in a controllable, pure DER system as reported in [1]. In a recent publication, Rong et al. [11] reported their 
experiment on the large enhancement of static yield stress of magnetorheological(MR) fluid under compression. They related this effect to the observed column size enlargement after compression under magnetic field. Based on the calculation and discussions given here, we believe that the magnetic force is not very sensitive to the structure. In any ER and MR experiment, the observed static yield stress of ER fluids are contributions both from electrostatic(or magnetic) interactions and other sources. When spheres are tightly packed by compression under magnetic field, the dry friction may play a very important rule in the static yield stress.

In this work we discussed two extreme conditions, the infinite column state and the chain state. While ER fluids with thick irregular columns arranged randomly is the observed situation in most experiments, which may be viewed as intermediate to the two cases studied here. Since our results for the two extreme cases are very close to each other in a large range of other parameters, it is a good approximation to use both cases in explaining experiment results of DER fluids. This work is supported by The Research Fund for the Doctoral Program of Higher Education and National Natural Science Foundation of China. We thank the referee to point out reference 11 .

[1] H. Ma, W. Wen, W. Y. Tam, and P. Sheng, Phys. Rev. Lett., 77,2499(1996).

[2] T.C Halsey and W.Toor, Phys. Rev. Lett., 65,2820 (1990); T.C. Halsey, Science, 258, 713 (1992).

[3] R. Tao. and J. M. Sun, Phys. Rev. Lett. 67, 398 (1991).

[4] H. Ma, W. Wen, W. Y. Tam, and P. Sheng, Advances in Physics, submitted.

[5] L. C. Davis, Appl. Phys. Lett., 60, 319(1992).

[6] J. M. Ginder and L. C. Davis, Appl. Phys. Lett., 65, 3410(1994).

[7] R. Tao, Qi Jiang, and H. K. Sim, Phys. Rev., E52, 2727(1995).

[8] H. J. H. Clercx and G. Bossis, Phys. Rev., E48, 2721(1993).

[9] D.J. Bergman, in Solid state physics, edited by h. Ehrenreich and D. Turnbull (Academic Press, New York, 1992), Vol.46, p.147; G.W. Milton, Appl. Phys. A 26,1207(1981); G. W. Milton, J. Appl. Phys. 52,5286(1980).

[10] J. C. M. Garnett, Philos. Trans. R. Soc. London 203,385 (1904); ibid 205, 237 (1906). 
[11] Y. Rong, R. Tao and X. Tang, Int. J. Adv. Manuf. Technol., 16, 822(2000). 